\title{
PBS-Free Polarization-Independent PON Coherent Receiver
}

\author{
Mario Rannello ${ }^{\circledR}$, Marco Presi ${ }^{\circledR}$, and Ernesto Ciaramella ${ }^{\circledR}$, Senior Member, IEEE
}

\begin{abstract}
We introduce a coherent receiver that, without DSP and/or polarization coding at the transmitter, requires no Polarization Beam Splitter and still is polarization-independent. It is experimentally demonstrated at $10 \mathrm{Gbit} / \mathrm{s}$ with a sensitivity of $-37.6 \mathrm{dBm}$ at FEC level. It can also be operated at higher bit-rates and finds application in high-speed access and datacenter networks.
\end{abstract}

Index Terms-Optical access network, datacenter fiber networks, coherent detection.

\section{INTRODUCTION}

$\mathbf{H}$ IGH-performance access networks require the development of high-sensitivity coherent detectors, at affordable cost. To make coherent detection compatible with this key requirement, the coherent receiver should have a simplified optical front-end and a DSP-free signal processing back-end [1]. Various simplified front-ends were considered for access network applications. They exploit in general configurations based on $2 \times 2$ or $3 \times 3$ optical couplers, which are simpler and cheaper components. A receiver based on the $3 \times 3$ coupler can be polarization-independent without the need of any polarization diversity [1]-[3], i.e., without doubling the front-end components [4]. As an alternative, polarization independence can be achieved with a single-polarization receiver, combined with Alamouti coding; here the complexity is moved to the transmitter, to realize the polarization coding [5], [6]. Another relevant feature is Digital Signal Processing (DSP); DSP is widely used in long-haul systems, but may not be needed in access networks, thus reducing cost and power consumption. Various simplified DSP-free solutions have been demonstrated for access networks; most of them are based on the Glance receiver [4], [7] or on the COCONUT polarization-independent schemes [3], [8].

A common feature of these receivers is that they need a Polarization Beam Splitter (PBS), which is an expensive block, and it is complex in photonic-integrated implementations. Therefore, a polarization-independent coherent receiver that needs no PBS would be a very interesting option [9].

Manuscript received July 7, 2020; revised August 24, 2020; accepted September 1, 2020. Date of publication September 9, 2020; date of current version September 29, 2020. (Corresponding author: Mario Rannello.)

Mario Rannello and Ernesto Ciaramella are with the Institute of Communication Information and Perception Technologies (TeCIP), Scuola Superiore Sant'Anna, 56124 Pisa, Italy (e-mail: mario.rannello@ santannapisa.it).

Marco Presi was with Scuola Superiore Sant'Anna, 56124 Pisa, Italy. He is now with Aerospazio Tecnologie, 53040 Rapolano Terme, Italy.

Color versions of one or more of the figures in this letter are available online at http://ieeexplore.ieee.org.

Digital Object Identifier 10.1109/LPT.2020.3022975

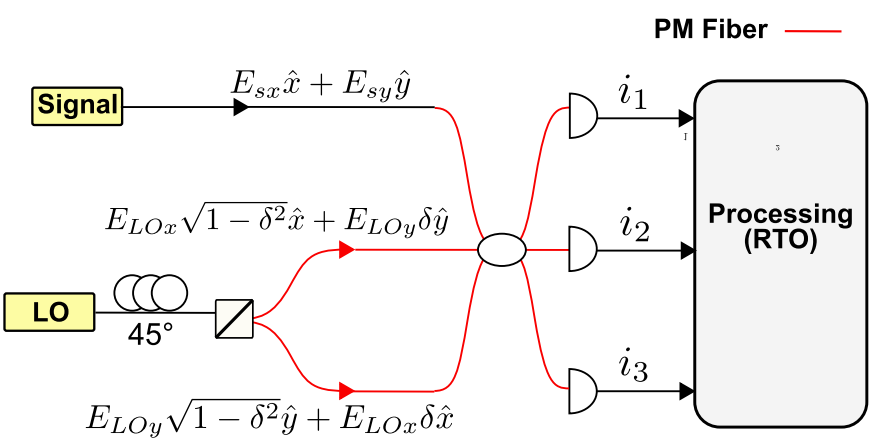

a)

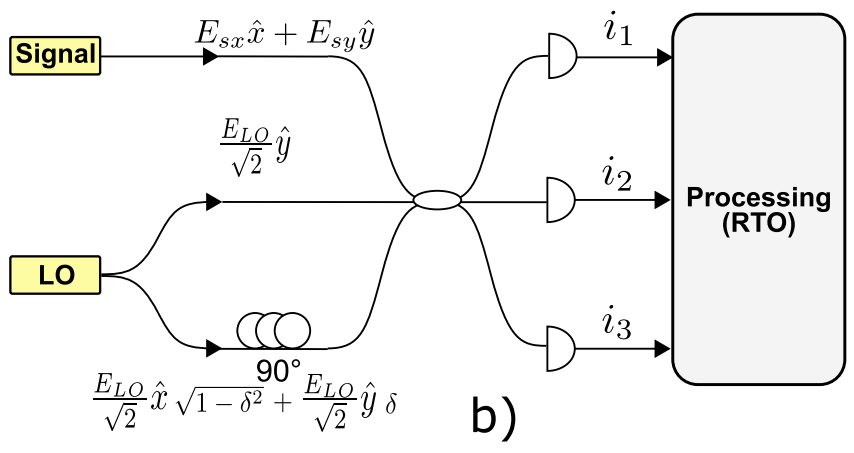

Fig. 1. Receiver schematics compared in this work. a) original scheme proposed in [2]. b): the PBS-free configuration introduced here. LO: Local Oscillator. Red lines indicate polarization maintaining optical fibers.

In this letter, we introduce for the first time a modification of one of the COCONUT polarization-independent receiver schemes, whose performances have been assessed in detail by means of numerical simulations [2], from which the PBS is successfully removed. In one of the configurations originally proposed, the PBS divides the Local Oscillator (LO) light into two orthogonal polarization states. However, its performance was impaired by the limited Polarization Extinction Ratio (PER) of the PBS. Here we replace the PBS by a simple 1:2 coupler and overcome the issues due to PBS employment. The system is demonstrated experimentally and discussed at $10 \mathrm{~Gb} / \mathrm{s}$.

\section{Operating Principle}

We introduce the new receiver starting from the known scheme that includes the PBS [2] and is shown in fig. 1-a. In this scheme the LO lightwave is split by the PBS into two orthogonal components, of same amplitude, which are 
coupled with the input signal into the coupler. The main issue associated with this configuration is the self-beat interference due to the LO. Indeed, in an ideal system, the PBS has an infinite PER, the two components of the LO are perfectly orthogonal, and, when combined in the coupler, they originate no optical beat interference. However, a real PBS has finite PER (usually $<25 \mathrm{~dB}$ ); this induces non-negligible beating between the two LO components and thus deteriorates the received signal, as we will observe. Here we model the effect of a non-ideal PBS by introducing the parameter $\delta$ that takes into account the non perfect separation of the orthogonal components of the $\mathrm{LO}$ and the non perfect orthogonality of the two PBS outputs (see Fig. 1-a).

The alternative implementation of the receiver that we present here is shown in Fig. 1-b: we replace the PBS by a simple 1:2 splitter on the LO and then rotate the output polarization of one of the two outputs so that the State of Polarization (SOP) of the resulting components are orthogonal. By a fine polarization control, the beat interference can be significantly suppressed. Also in this case, we can take into account a non-perfect polarization rotation by means of the same quantity $\delta$. In both cases, in the expression of the three output currents $i_{k}$ we see the effect of the non-perfect orthogonality of the LO components at the input of the $3 \times 3$ coupler. In order to derive it, we write the signal as $\underline{E}_{s}=$ $E_{s x} e^{j \theta_{s x}} \hat{x}+E_{s y} e^{j \theta_{s y}} \hat{y}$ and assume that the LO is linearly polarized (i.e. $\underline{E}_{L O}=E_{L O x} \hat{x}+E_{L O y} \hat{y}$ ), the photocurrents $i_{k}$ are given by:

$$
\begin{aligned}
i_{k} \propto & \mid \underline{E}_{s} e^{j \frac{2(k+1) \pi}{3}}+E_{L O x} e^{j \frac{2 k \pi}{3}} \sqrt{1-\delta^{2}} \hat{x}+E_{L O y} e^{j \frac{2 k \pi}{3}} \delta \hat{y} \\
& +E_{L O y} e^{j \frac{2(k-1) \pi}{3}} \sqrt{1-\delta^{2}} \hat{y}+\left.E_{L O x} e^{j \frac{2(k-1) \pi}{3}} \delta \hat{x}\right|^{2} \\
= & \left|\underline{E}_{S}\right|^{2}+\left|E_{L O x}\right|^{2}+\left|E_{L O y}\right|^{2} \\
& +2 E_{S x} E_{L O x} \sqrt{1-\delta^{2}} \cos \left(\phi_{k x}\right) \\
& +2 E_{S y} E_{L O y} \sqrt{1-\delta^{2}} \cos \left(\phi_{k y}\right) \\
& +2 E_{S x} E_{L O y} \delta \cos \left(\phi_{k x}^{\prime}\right)+2 E_{s y} E_{L O x} \delta \cos \left(\phi_{k y}^{\prime}\right) \\
& +E_{L O x} E_{L O y} \delta \sqrt{1-\delta^{2}} \cos \left(\frac{2 k \pi}{3}\right)
\end{aligned}
$$

for $k=1,2,3$. This expression is valid for both the schemes previously presented, which are indeed formally equivalent. The only differene is that $\delta$ indicates the PBS non-ideality or the error in the rotation, in the two cases. While we address the reader interested in the mathematical details of the scheme operation to [2], let us now briefly describe the different contributions appearing in the expression of the currents.

In the equation above we have indicated with $\phi_{k x}, \phi_{k y}, \phi_{k x}^{\prime}$ and $\phi_{k y}^{\prime}$ the phase shift terms better defined below. Each $i_{k}$ contains different contributions, that are shown on different lines of the right-hand-side of the equation. First, the directdetection term of the signal has negligible amplitude (in the hypothesis of working in the sensitivity range, with low optical power detected), whilst the direct-detection of the LO can be easily suppressed by a DC-block. Secondly, we have the mixing terms between the received signals and the LO, which is the useful signal (second and third row or the right hand side), $\phi_{k x}$ and $\phi_{k y}$ are, respectively, the phase shifts between the $\mathrm{x}$ and $\mathrm{y}$ components of the signal and the LO). Then, on the fourth row, we see the terms due to the beating between the signal and the residual fraction of $\delta$ in the LO, which is not perfectly suppressed ( $\phi_{k x}^{\prime}$ and $\phi_{k y}^{\prime}$ indicate, similarly as before, the phase shifts between the corresponding components): they have negligible impact $\delta$ is sufficiently small. Again, major details on the explicit expression of these phase terms, that we do not report here, can be found in [2].

Finally, we see the beating term of the LO (LO-LO beating): this cannot be neglected and is the most critical, since it can be comparable to the useful signal even if $\delta \ll 1$. This is proven by comparing the typical amplitudes of the received signal (in the order of $-40 \div-30 \mathrm{dBm}$ ) and the LO power (typically around $+10 \mathrm{dBm}$ ). Using these values, even a high-end PBS with a high PER of $30 \mathrm{~dB}$ gives a LO-LO beating comparable with the LO-signal beating. In addition, inexact mechanical alignment of the PBS axes can also increase the impact of the LO-LO beating. Since it is the result of the product of the $x$ and y components of the LO, this has a noise spectrum that is very similar to the Relative Intensity Noise (RIN) of the LO laser, i.e. it extends from the DC to few GHz. In other receivers the RIN effect might be effectively suppressed by a balanced detection configuration (either physically [10] or by DSP [11]), however here the LO-LO beating term cannot be canceled by using a balanced configuration, since it appears with a different phase shift at each output. This feature prevented using the scheme in homodyne regime, so far. However, we note that this scheme could be used in heterodyne, with a high frequency detuning between the signal and the LO: in this case the useful term is at much higher frequency than the LO-LO beating, which can thus be suppressed by an proper high-pass filtering [3]. This solution, however, requires a much wider bandwidth of the components and is also not optimal in terms of the spectral efficiency.

In the implementation of Fig. 1-b, thanks to fine polarization control, the beat interference can be significantly suppressed. This allows for effective intradyne detection. In this case, a minor LO crosstalk is observed, which is due to the unpolarized ASE noise emitted by the LO laser. Noteworthy, this modified scheme does not require any expensive PBS, as in the original scheme of fig. 1-a), in favor of a simpler and cost-effective implementation. Of course, in a controlled lab environment, with short fiber pigtails, polarization is very stable; thus we can maintain the orthogonality condition during the time needed for the experiments. However, a realization of the scheme in integrated photonics would provide the fixed orthogonality of the two components, since the orientation of the electric field in a PIC does not change and is determined mainly by the geometry of the photonic waveguide.

\section{EXPERIMENT}

We use a simple setup to compare the different implementations of the Polarization Independent (PI) receiver, which is illustrated in fig. 2. The NRZ signal is obtained by a $10 \mathrm{~Gb} / \mathrm{s}$ external modulation of a Distributed Feedback Laser (DFB) laser $\left(\lambda=1542 \mathrm{~nm}\right.$, optical power $P_{S}=13 \mathrm{dBm}$, RIN $=$ $-143 \mathrm{~dB} / \mathrm{Hz}$, linewidth $\Delta v=10 \mathrm{MHz}$ ). The modulator 


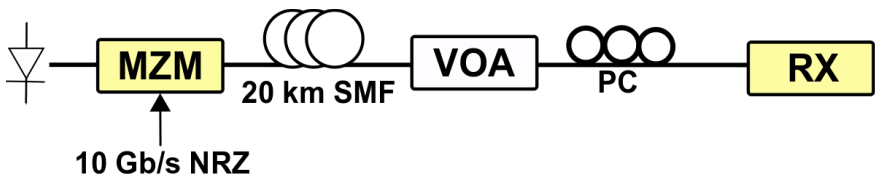

Fig. 2. Experimental setup. RX can be any of the two configurations of Fig. 1.
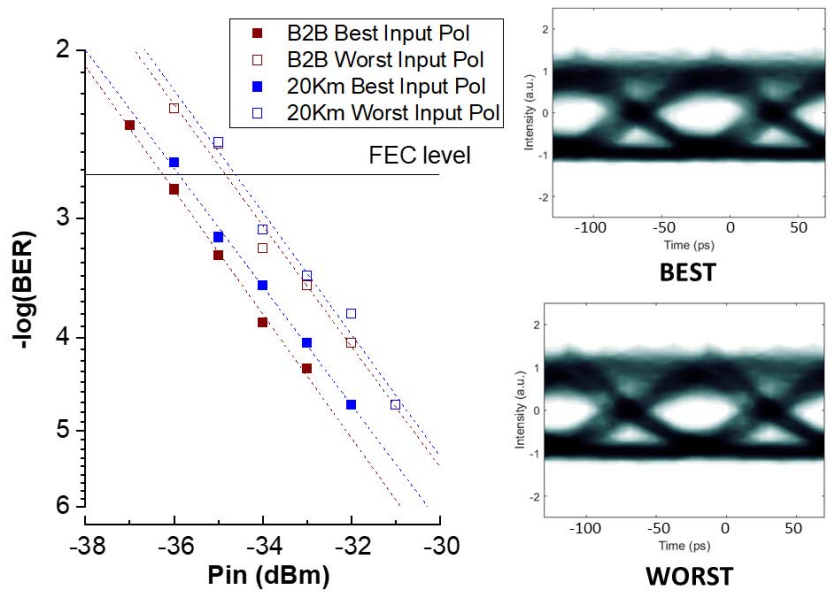

Fig. 3. Performance of PBS-based RX of Fig. 1-a. Eye diagrams are taken with a received optical power of $-30 \mathrm{dBm}$.

is a Mach-Zehnder Modulator (MZM) (electrical bandwidth of $40 \mathrm{GHz}$ ). The signal is then propagated over $20 \mathrm{~km}$ of standard single mode fiber ( $\mathrm{D}=16 \mathrm{ps} / \mathrm{nm} / \mathrm{km}, \alpha=0.2 \mathrm{~dB} / \mathrm{km})$, and passes then a Variable Optical Attenuator (VOA) and a Polarization Controller (PC), before detection. The presence of the $\mathrm{PC}$ on the signal branch is due to the necessity to obtain the 'best' and 'worst' operating condition of the receivers. In fact, the 'best input polarization' corresponds to the case where the input polarization of the signal is aligned to one of the two principal axes of the $3 \times 3$ coupler. On the other hand, the 'worst input polarization' corresponds to the case in which the input polarization has an angle of $45^{\circ}$ with both the principal axes of the coupler, as explained in detail in [2].

In both the configurations, the LO is an External Cavity Laser ( $\mathrm{C}$ band, detuning with respect to the signal $\Delta v=$ $9 \mathrm{GHz}$ ). In the receiver of Fig. 1-a the LO output light passes through a Variable Optical Attenuator (VOA), a $45^{\circ}$ polarization rotator and then is injected into an high quality PBS, having $30 \mathrm{~dB}$ PER and an insertion loss of $1.5 \mathrm{~dB}$. The PBS has polarization maintaining (PM) output fibers that are connected to a $3 \times 3$ coupler, also with PM fiber pigtails (and insertion loss of $0.6 \mathrm{~dB}$ ). The signal is injected into the third port of the coupler. In the novel implementation (Fig.1-b), the LO light enters first the VOA, then a $3 \mathrm{~dB}$ optical splitter (with SMF pigtails and insertion loss of $0.2 \mathrm{~dB}$ ) and finally a second $3 \times 3$ optical coupler with common SMF pigtails (i.e. without PMF). Also this second $3 \times 3$ coupler has an insertion loss of around $0.6 \mathrm{~dB}$. One of the two LO lightwaves passes through a PC before the $3 \times 3$ coupler. In the first implementation, the polarization orthogonality should be ensured by the use of PMF components. In the second, the orthogonality is obtained by minimizing the self-beat interference of the LO
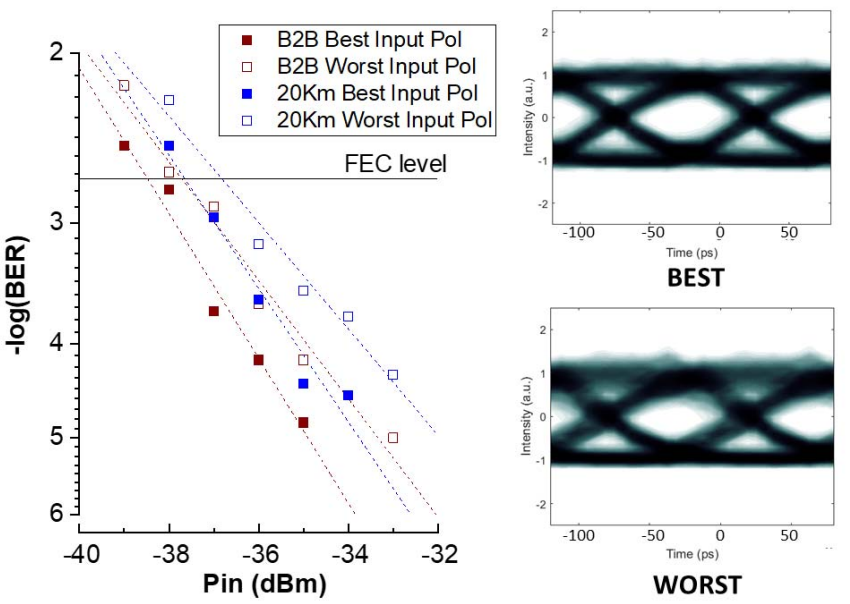

Fig. 4. Performance of novel proposed PBS-free configuration (see Fig. 1-b). Eye diagrams are taken with a received optical power of $-30 \mathrm{dBm}$.

at the receiver output, by means of the PC, which is carefully adjusted so that the two LO components arrive with orthogonal SOP at the photodiodes.

In all the experiments, the $\mathrm{LO}$ output power is set to around $+12 \mathrm{dBm}$, thus it arrives at both the inputs of the $3 \times 3$ coupler with the same power $(+9 \mathrm{dBm})$. The effects of varying LO power and LO RIN noise on the performance were investigated in [2]. For both the recevier implementations, the same three identical single-ended photodiodes with $15 \mathrm{GHz}$ E/O bandwidth are used and signal processing reconstructs the NRZ signal off-line in a $40 \mathrm{GSa} / \mathrm{s}$ Real-Time Oscilloscope (RTO) of $13 \mathrm{GHz}$ analog bandwidth, by squaring and adding the three $i_{k}[8]$.

We characterized the performance of the two receiver implementations by means of BER measurements and eye diagrams. In the case of the coupler-based receiver, we first minimized the self-beating due to the LO by acting on the PC shown in Fig. 1-b. The minimization was done in two steps: first without any input signal to the receiver, the self beating was visualised on the RTO and roughly minimized in amplitude. In a second step, the modulated signal was turned on and the BER measurement was done, a fine tuning was done by minimizing the BER, which was provided almost in real-time by the routine running on the oscilloscope. So the condition of orthogonality was reached when the measured BER, with all the other parameters on the setup fixed, was at its minimum. This adjustment was carried out with a signal power close to the receiver sensitivity, when a stable BER value was provided by the routine in a quite short time.

In both cases, BER measurements were taken for best and worst operating conditions of the signal at the receiver. The "best" and "worst" operating conditions were obtained by manual SOP adjustment, acting on the PC placed in front of the receiver, and shown in Fig. 2, minimizing (or maximizing) the eye closure, constantly monitored on the Real-Time Oscilloscope. In Fig. 3 we report the performance of the PBS-based configuration. In Fig. 4 we report, instead, the performance of the novel PBS-free configuration proposed here (Fig. 1-b), making use of a coupler on the LO branch. As can be seen, the PBS-based receiver has a sensitivity in B2B at 
the FEC level $\left(\mathrm{BER}=2 \times 10^{-3}\right)$ between $-36.2 \mathrm{dBm}$ (best input polarization) and $-34.7 \mathrm{dBm}$ (worst input polarization), while the novel coupler-based $\mathrm{Rx}$ is between $-38.5 \mathrm{dBm}$ and $-37.6 \mathrm{dBm}$. We see a relevant improvement (between $2.3 \mathrm{~dB}$ and $2.9 \mathrm{~dB}$ ). This improvent can be ascribed in part to the insertion loss gain (of around $1.3 \mathrm{~dB}$, in our case), in part to the fine control of the LO-LO beat that we managed to achieve with the coupler configuration. This gain is inevitably linked to the particular components in use, yet we can see that this feature has some generality. Indeed, the insertion loss of a PBS is typically higher than the one of a fiber splitter (or also a splitter realised in integrated photonics technology), given the current technological development. Moreover, the $\delta$ parameter, in the case of the PBS scheme, is linked to the physical realization of the PBS, whilst a potential realization of the coupler-based scheme in integrated photonics would provide the fixed orthogonality of the two components of the LO, as long as the fabrication process is able to control with sufficient accuracy the geometry of the waveguide. Moreover, the sensitivity gap between 'best' and 'worst' polarization cases decreases from around $1.5 \mathrm{~dB}$ in the case of the PBS-based version, to $0.9 \mathrm{~dB}$ in the case of the coupler-based one. This confirms that replacing the PBS can also give a performance improvement, both in terms of sensitivity and robustness to polarization variations. With respect to the performances after $20 \mathrm{~km}$ transmission, the PBSbased receiver has a sensitivity between $-35.9 \mathrm{dBm}$ and $-34.6 \mathrm{dBm}$, while the novel coupler-based $\mathrm{Rx}$ is between $-37.6 \mathrm{dBm}$ and $-36.8 \mathrm{dBm}$. In this case, the improvement is between 1.7 and $2.9 \mathrm{~dB}$. The reduction of the improvement is due to the fact that with the novel configuration we observe a slightly higher penalty upon transmission (around $0.8 \mathrm{~dB}$ ) with respect to the PBS configuration (around $0.3 \mathrm{~dB}$ ). This can be observed by comparing the BER curves in Fig. 3 and 4. Further investigation regarding this effect should be carried out, but in any case we believe this does not impact significantly on the validity of the proposed scheme. Moreover, we observe a slightly different slope between best and worst case polarization in Fig. 4. This actually seems just an effect of numerical interpolation of the data, combined with the higher relative error affecting low BER measurements. The script of BER calculation required a long measurement time, in the order of minutes, at low BER values (in the range $10^{-5}-10^{-6}$ ), for the accumulation of a statistically relevant number of errors, and in any case those measurements suffer from an higher relative error than to BER values measured close to the sensitivity of the receiver (BER around $10^{-3}$ ).

In both cases we report also the eye-diagrams taken after $20 \mathrm{~km}$ of propagation, for the best and worst polarization: a sensible improvement can also be appreciated in terms of the eye opening. Eye diagrams with a received optical power of $-30 \mathrm{dBm}$.

\section{CONCLUSION}

We experimentally investigated the PBS-on-LO scheme of the Polarization-Independent receiver [2]. We found that high-quality PBS (with very high extinction ratio) is needed to operate the receiver correctly, which cannot be simply provided even by commercial high-end PBS devices. This is due to the fact that any commercial component on the LO would result into relevant beating noise in our receiver; therefore we proposed and demonstrated an alternative approach, based on a passive coupler instead of a PBS; the scheme effectively suppresses the beating noise and allows for polarization independence without a PBS element, leading to $-37.6 \mathrm{dBm}$ sensitivity at FEC value. This scheme has an added value for implementation, especially considering future prospects of photonic integration.

\section{REFERENCES}

[1] V. Houtsma and D. van Veen, "Bi-directional 25G/50G TDM-PON with extended power budget using 25G APD and coherent detection," J. Lightw. Technol., vol. 36, no. 1, pp. 122-127, Jan. 1, 2018.

[2] E. Ciaramella, "Assessment of a polarization-independent DSP-free coherent receiver for intensity-modulated signals," J. Lightw. Technol., vol. 38, no. 3, pp. 676-683, Feb. 1, 2020.

[3] J. Tabares, V. Polo, and J. Prat, "Polarization-independent heterodyne DPSK receiver based on $3 \times 3$ coupler for cost-effective udWDM-PON,' in Proc. Opt. Fiber Commun. Conf. Exhib. (OFC), Mar. 2017, pp. 1-3.

[4] J. A. Altabas et al., "Real-time 10Gbps polarization independent quasicoherent receiver for NG-PON2 access networks," in Proc. Opt. Fiber Commun. Conf. Washington, DC, USA: Optical Society America, 2018, pp. Th1A-3.

[5] M. S. Erkilinc et al., "Polarization-insensitive single-balanced photodiode coherent receiver for long-reach WDM-PONs," J. Lightw. Technol., vol. 34, no. 8, pp. 2034-2041, Apr. 15, 2016.

[6] I. C. Valadez, A. Lerín, V. Polo, and J. Prat, "Flexible D (Q) PSK 1.25-5 Gb/s UDWDM-PON with directly modulated DFBs and centralized polarization scrambling," in Proc. Eur. Conf. Opt. Commun., Sep./Oct. 2015, pp. 1-3.

[7] M. S. Erkilinc et al., "Comparison of low complexity coherent receivers for UDWDM-PONs ( $\lambda$-to-the-user)," J. Lightw. Technol., vol. 36, no. 16 , pp. 3453-3464, Aug. 15, 2018.

[8] M. Artiglia, M. Presi, F. Bottoni, M. Rannello, and E. Ciaramella, "Polarization-independent coherent real-time analog receiver for PON access systems," J. Lightw. Technol., vol. 34, no. 8, pp. 2027-2033, Apr. 15, 2016.

[9] I. Molina-Fernandez et al., "Coherent receivers for demanding applications," in Proc. Opt. Fiber Commun. Conf., 2016, pp. 1-3.

[10] E. Patzak and R. Lancenhorst, "Sensitivity degradation of conventional and balanced $3 * 3$ port phase diversity DPSK receivers due to thermal and local oscillator intensity noise," Electron. Lett., vol. 25, no. 8, pp. 545-547, 1989.

[11] C. Xie et al., "Colorless coherent receiver using $3 \times 3$ coupler hybrids and single-ended detection," Opt. Express, vol. 20, no. 2, pp. 1164-1171, 2012 . 